OPEN ACCESS

Edited by:

Jeremy N. Marchant-Forde, Livestock Behavior Research Unit (USDA-ARS), United States

Reviewed by: Lindsey Eve Hulbert, Kansas State University, United States Luiz Carlos Pinheiro Machado Fo, Federal University of Santa Catarina, Brazil

*Correspondence:

Patricia Tatemoto patricia.tatemoto@usp.br

Specialty section: This article was submitted to Animal Behavior and Welfare, a section of the journal Frontiers in Veterinary Science

Received: 19 June 2018 Accepted: 21 October 2019 Published: 19 November 2019

Citation:

Tatemoto P, Bernardino T, Alves $L$ and Zanella AJ (2019) Sham-Chewing in Sows Is Associated With Decreased Fear Responses in Their Offspring. Front. Vet. Sci. 6:390 doi: 10.3389/fvets.2019.00390

\section{Sham-Chewing in Sows Is Associated With Decreased Fear Responses in Their Offspring}

\author{
Patricia Tatemoto*, Thiago Bernardino, Luana Alves and Adroaldo José Zanella
}

Department of Veterinary Medicine and Animal Health, Center for Comparative Studies in Sustainability, Health and Welfare, School of Veterinary Medicine and Animal Science, FMVZ, University of São Paulo, São Paulo, Brazil

We hypothesized that sham-chewing expressed by the dam during gestation affects fetus programming. The goal of this study was to assess the effects of maternal sham-chewing on offspring welfare indicators, such as behavior and physiology. Sows that exhibited consistent sham-chewing on at least two of 6 days of observation $(N=7)$ were compared with sows that had never performed sham-chewing (non-sham-chewing sows; $N=4$ ) during these 6 days. Salivary samples from sows and piglets were collected and cortisol concentrations were analyzed to assess the hypothalamic pituitary adrenal (HPA) axis activity as cortisol is a physiological indicator of welfare. Moreover, placental tissue was collected, right after farrowing, to assess cortisol and cortisone concentration. Piglet behavior and fear tests were performed after weaning (one couple per sow). In the fear tests, data was collected in an open field test to determine the states of fear indicators. Non-sham-chewing sows had lower concentrations of cortisol on days 91 and 92 of gestation in the morning. In addition to this, placental cortisol was higher among sham-chewing sows than non-sham-chewing sows. In the open field test, piglets born from non-sham-chewing sows demonstrated more latency to move in the arena and less activity, indicating more fear. Based on our data, we concluded that the expression of maternal sham-chewing is related to less fear in their offspring. Although stereotypies have been studied, attention has not been devoted to the effects of the prenatal period in considering a fetal reprogramming approach.

Keywords: cortisol, cortisone, gestation, prenatal stress, placenta, stereotypies

\section{INTRODUCTION}

Stereotypic behaviors or "stereotypies" are a wide range of repetitive and apparently functionless patterns that often develop in environments that likely contribute to poor animal welfare (1). This behavior often develops in animals housed in environments with few stimuli, or that involve physical restraint(s), fear, and/or frustration (1). Stereotypic behaviors is also affected by a genetic component (2) and personality predisposition $(3,4)$. Stereotypies may also be an inherited pattern for some species, e.g., courtship. Stereotypies in general have a multifactorial cause, in which there is a synergetic effect of internal and external stimulus triggering their expression. However, some variables have a greater impact on the triggering of stereotypic behavior than others when the diverse range of environmental factors are considered (5). 
On this study, we considered only the stereotypy shamchewing. Oral stereotypies have been discussed as a beneficial strategy against gastrointestinal acidity (6). Researchers suggests that sham-chewing is related to foraging and other natural behaviors (6-8). The behavioral sequences of sham-chewing could be as a response to frustration for natural foraging (6) or just the appetitive behavior without the consummatory phase that would be the manipulation of food.

Stereotypic behavior is often considered to be an indicator of welfare $(1,9,10)$ because behaviors provide clues about psychological states that are difficult to assess. Therefore, shamchewing can be considered an indicator of animal welfare. However, stereotypies are sometimes not consistent with cortisol levels (11), a physiological welfare indicator involved in the stress cascade $(12,13)$. Some researchers propose, based on the "Coping Hypotheses," that the performance of stereotypies reduces distress $(14,15)$.

However, it remains unclear how the long-term effects of stereotypies influence phenotypes in offspring. In mammals, pregnancy has an important role in shaping the organism as the dam's environment may influence the offspring development during gestation. This concept has emerged from the "thrifty phenotype hypothesis," in which neurodevelopment programming induces alterations to cope with challenges in anticipating the postnatal environment (16). In other words, the prenatal environment has the potential to affect the offspring phenotype and prepare individuals for the environment that they will be inserted into in order to prepare them to better cope with challenges. The environment in which an animal is maintained during gestation may result in changes in several offspring parameters (17-22). By this mechanism, factors such as emotional reactivity, responsiveness to stressors, and cognition can be modulated by challenges in the prenatal and neonatal periods $(21,23,24)$. It was showed before that some stressors, such as negative interactions with the handler $(21,22,25)$ and social stress (21), can alter emotional reactivity, social behavior, and responsiveness to stressors, cognition, and memory in the offspring. Moreover, offspring of sows that experienced less hunger during gestation have exhibited reduced aggressive behavior prior to weaning (26).

Although some studies have demonstrated that stress during pregnancy or prenatal stress can generate changes that are not necessarily pathological (17), offspring exposure to an excess of glucocorticoids affect important brain structures and lead to negative effects $(21,22,25)$. Glucocorticoids are important stress hormones in adult animals, but their functions are more diverse in the fetus, and their effects may be completely different depending on gestational age and the severity and duration of the exposure (27).

The placenta has a role in protecting the fetus in the prenatal period and, therefore, modulates stressful events experienced by the maternal organism and acts as a buffer (28). In mammals, $11 \beta$-hydroxysteroid dehydrogenase enzyme type 2 (11ßHSD2) in the placenta forms a barrier that protects the fetus from high levels of maternal cortisol because it oxidizes the biologically active form of cortisol in cortisone $(28,29)$. Chronic stressful situations have the potential to inhibit the capacity to upregulate type 2 enzyme activity, and the capacity to adapt placental
$11 \beta$ HSD2 is significantly reduced by previous exposure to chronic stress (30), thus reducing the protective capacity of the placenta.

The effects of prenatal stress increase cortisol concentration, which can cross the placenta and affect brain structures such as the hippocampus and amygdala, and may generate changes in the offspring's emotionality $(31,32)$. Exploratory and fearrelated behaviors such as activity and vocalizations can be quantified from novel object and open field tests (33-36). Our goal was to evaluate the consequences of a stereotypic behavior (sham-chewing) in sows during gestation in the physiological and behavioral parameters of their offspring. In this sense, if the expression of sham-chewing is helping the sows to cope with stress, it should potentially affect fetus programming. The hypothesis is that sham-chewing expressed by the mother during gestation affects fetus programming.

\section{MATERIALS AND METHODS}

\section{Animals and Housing Conditions}

The study was carried out at the Araporanga Farm, at Jaguariaíva, Paraná, Brazil. This study was conducted according to the ethical principles of animal experimentation and under the approval of the Ethics Committee on Animal Use of the School of Veterinary Medicine and Animal Science (University of São Paulo, Brazil), protocol number 6157201114. Authors ensured that the manuscript conforms to the ARRIVE Guidelines for Reporting Animal Research.

The analysis was performed on 11 pregnant sows (TopGen Afrodite $\left.{ }^{\circledR}\right)$, from a total of 30 sows, and distributed according to a body condition score in three conventional pens with a concrete floor (10 sows per pen, in which only six were considered before the inclusion criteria). The farm staff previously made the distribution according to body conditions, considering the size and weight of the sows. The sows were from the 2 nd to 7 th parity order ( $T$-test; $p>0.05$ ). We compared four sows that had never exhibited sham-chewing (non-sham-chewing sows) with seven that consistently exhibited sham-chewing (on at least 2 of 6 days of observation) and divided them into three pens with mixed treatment.

The feed was offered twice daily, at 07:00 and 11:40 a.m., and the animals had ad libitum access to water. Each pen was $6 \mathrm{~m}$ long $\times 3.86 \mathrm{~m}$ wide with a solid and slatted concrete floor area of $3.97 \mathrm{~m}$ in length, and the pen walls were $0.85 \mathrm{~m}$ high. The feeder was $5 \mathrm{~m}$ long and $0.37 \mathrm{~m}$ wide.

The piglets were weaned at 28 days of age, vaccinated (vaccines against porcine circovirus, Streptococcus suis, Haemophilus parasuis, and Mycoplasma hyopneumoniae) and transported from the Araporanga Farm in Jaguariaíva-PR (where the first stage of the experiment was performed) to the Fernando Costa Campus of the University of São Paulo in Pirassununga-SP, which involved approximately $8 \mathrm{~h}$ of travel. One couple of piglets per sow was randomly selected for the second part of the experiment. During the trip, each couple was placed in a transport box $(73.5 \mathrm{~cm}$ long $\times 53 \mathrm{~cm}$ wide $\times 21 \mathrm{~cm}$ high), making it impossible for aggressive interaction between different litters, and these were lined with straw (hay). 
After weaning, the piglets were housed in suspended pens, with 6 litters per pen. Each pen consisted of 12 animals, a couple from each sow. We had three pens and the animals were grouped according to their mother's group (mixed treatment). The piglets had ad libitum access to food and water.

\section{Experimental Design}

To assess the effects of sham-chewing on the offspring during gestation, we studied the behavior and salivary cortisol concentration from their piglets. The behaviors evaluated included aggressiveness, nosing, and fear-related behaviors. In addition, the glucocorticoids in the placental tissue were accessed.

\section{Sow Behavioral Data}

To collect behavioral data, an ethogram was adapted (37) and summarized in Table 1. Behavioral measures of sows were obtained by direct observation on days $88,89,91,92,106$, and 107 , which represent the final one-third of the gestational period. The collection periods were conducted over two consecutive days to avoid possible interference by stressful events. The behavioral assessments were performed by direct observation at 17:30. Each animal was observed three times per uninterrupted $120 \mathrm{~s}$ period, totaling 6 min per animal per observation time, which, in the 6 days of observation, totaled $36 \mathrm{~min}$ per animal. Two observers were previously standardized to avoid bias in data collection. Observations were performed using a combination of methods for behavioral measures, which started with a scan sample, followed by a focal animal with continuous observation (uninterrupted $120 \mathrm{~s}$ ).

\section{Salivary Cortisol and Enzyme-Linked Immunosorbent Assay}

Saliva collection was performed on the same days of the behavioral evaluation, on days $88,89,91,92,106$, and 107 so as to correspond to the gestation length. Two samples were collected per animal, at 06:00 a.m. and 18:00 p.m., to follow the circadian rhythm of cortisol, and the effect of sham-chewing on the hypothalamic pituitary adrenal (HPA)-axis activity. The saliva was collected using hydrophilic cotton on two rollershaped units tied to dental floss with long tips that were presented to each animal. The animal chewed the cotton until it was saturated with saliva. The first sample collected was discarded; the collection was repeated to collect only recently produced saliva. Once the second sample was collected, it was placed into a $15 \mathrm{~mL}$ tube. Subsequently, the tube was packed in an icebox until the end of the collection, and the sample was frozen at $-20^{\circ} \mathrm{C}$ until processing. Thawing was performed in a box containing ice in a temperature-controlled room. After complete thawing, the sample was centrifuged for $10 \mathrm{~min}$ at $1,000 \times \mathrm{g}$; the supernatant was aliquoted into microtubes and frozen again at $-20^{\circ} \mathrm{C}$ until analysis. This process assisted the removal of mucins and other components that may have interfered with the analysis. For sample analysis, $50 \mu \mathrm{l}$ of saliva was analyzed with a cortisol enzyme immunoassay [EIA-based on (38)] in duplicate for each sow, with a pool of each gestation period, without mixing the morning and afternoon collections (e.g., with samples from 88
TABLE 1 | Definition of behaviors for data collection of pregnant sows.

\begin{tabular}{ll}
\hline Behavior & Definition \\
\hline Sleep & $\begin{array}{l}\text { Eyes closed, lying ventrally or laterally, no ears } \\
\text { movements } \\
\text { Lying with the belly facing the ground with all the limbs } \\
\text { under the body } \\
\text { Lying ventrally }\end{array}$ \\
$\begin{array}{l}\text { Lying with all the members extended laterally in one side } \\
\text { Standing }\end{array}$ & $\begin{array}{l}\text { Body supported by the four limbs } \\
\text { Continuous chewing without the presence of visible } \\
\text { food in the oral cavity }\end{array}$ \\
Rooting the floor & $\begin{array}{l}\text { Snout touches the ground followed by head movements } \\
\text { Licking the floor }\end{array}$ \\
Tongue touches the floor and is followed by movements \\
with the head \\
Interacting fence or gate
\end{tabular}

The caption E indicates behaviors that were measured (only the events and not the duration).

and 89 gestation days in the morning collection). The sensitivity of the EIA was $0.2 \mathrm{pg} /$ well.

\section{Farrowing}

Sows were moved to the farrowing crates at 108 days of gestation. The deliveries were monitored and occurred in conventional farrowing crates. At birth, each piglet had its umbilical cord tied with string kept in an antiseptic solution and dipped in iodine $(10 \%)$ for $5 \mathrm{~s}$. The piglets were then cleaned with paper towels, assigned a number for the order of birth on their back with a stick marker, and passed through antiseptic powder to reduce body moisture. After this initial management, the piglets were placed to ingest colostrum. On the first day of life, the piglets had their teeth ground, tail docked, ears notched, and individual weight recorded. Dextran iron supplementation was administered the day after delivery.

\section{Placenta Collection and Glucocorticoid Analysis}

The placenta was collected from four piglets per sow, in which on standardized (size and location) piece from each placenta was cut and subsequently frozen in a $-20^{\circ} \mathrm{C}$ freezer. All placentas from each sow were macerated together to prepare a pooled sample. Once the homogenized placenta was powder-like, $0.1 \mathrm{~g}$ was placed in a $1.5 \mathrm{~mL}$ microcentrifuge tube and $200 \mu \mathrm{L}$ of ultrapure water was added; the suspension was vortexed for $15 \mathrm{~s}$. Subsequently, $20 \mu \mathrm{L}$ was placed in another similar tube for total protein analysis [performed in triplicate for each sample, using the Bradford protocol (39)]. One milliliter of ethyl acetate was added to the tube with water and placenta, vortexed for $15 \mathrm{~s}$, and centrifuged for $15 \mathrm{~min}$ at $4^{\circ} \mathrm{C}$. The supernatant $(400 \mu \mathrm{L})$ was transferred to a new $1.5 \mathrm{~mL}$ microtube; the second (duplicate) 
was transferred to another tube. All samples were dried overnight in a fume hood until all the liquid volume evaporated. For glucocorticoid analysis, all samples were re-suspended in the same volume using assay buffer. The analysis was performed using the same EIA protocol for salivary cortisol (see section Salivary Cortisol and Enzyme-Linked Immunosorbent Assay). The cortisone analysis used specifically first antibody and biotin.

\section{Piglet Behavioral Data}

Throughout the experimental period, each pen was monitored by video cameras (Seco Infrared Domeball CCD Sony ${ }^{\circledR}$, lens $3.6 \mathrm{~mm}$, Case IP66) for further analysis of behaviors, such as aggressiveness and nosing. In this study, nosing was considered in any part of a pen mates' body (duration) and agonistic interaction (duration). Agonistic interactions have been characterized for pushing, head-knocking, biting, and chasing (40-42). This study considered only the behaviors made indubitable by video recording. The duration of each agonistic interaction considered started and ended with one of the cited behaviors.

\section{Exploratory and Fear-Related Tests}

A combination of open field and novel object tests (43) was performed to assess fear behavioral indicators or the exploratory motivations of each animal. The tests were conducted in all piglets at 41 days of age. The piglets were tested individually, and we returned then to the pen immediately after the test. The combination of tests enabled a previous piglets' habituation in the arena test, in which the open field test preceded the novel object test. The animals were tested in the arena $(243 \times 194.5 \mathrm{~cm})$, which contained demarcations on the ground forming quadrants. The duration of each test was $5 \mathrm{~min}$, totaling $10 \mathrm{~min}$. The piglets were gently placed in a predetermined location in the arena and they were recorded during the test period. To record the behavior, we used IP video cameras (Seco Infrared Domeball CCD Sony ${ }^{\circledR}$, lens $3.6 \mathrm{~mm}$, Case IP66). The definition of the analyzed behaviors can be found in Table 2. The latency to walk was quantified, as well as the number of central and lateral quadrants assessed, walking time (activity), freezing time, and vocalizations (events). After this test, a novel object was inserted (traffic cone) using a pulley system in the center of the pen. Subsequent behaviors were recorded for $5 \mathrm{~min}$. In this test, the latency for walking, time near the object (quadrants surrounding the object), time exploring the object (near the object with the head facing the object), freezing time, and vocalization (events) were evaluated. After each animal

TABLE 2 | Definition of behaviors for fear tests in piglets.

\begin{tabular}{ll}
\hline Behavior & Definition \\
\hline Latency to walk & Duration to start to walk \\
Number of quadrants & Quadrants accessed in numbers \\
Activity & Duration of walking to any direction \\
Freezing time & Duration spent without any movements \\
Vocalizations & Number of vocalizations \\
Near to object & Duration in quadrants (eight) surrounding the cone
\end{tabular}

was tested, the pen was washed with water to reduce possible chemical clues, as well as to remove feces and urine. The pen was also washed before the start of the first test of the day to standardize the entry of piglets in the arena test.

\section{Saliva Collection From Piglets}

Similar to the sows, saliva collection in piglets aimed to assess the activity of the HPA axis in relation to cortisol. The samples were collected on days $28,29,35$, and 36 , with two samples collected individually at 06:00 a.m. and 18:00 p.m. The collection of saliva was performed using the same methodology used for the sows (see section Salivary Cortisol and Enzyme-Linked Immunosorbent Assay). As these were piglets and did not produce significant amounts of saliva, the first cotton swabs were considered, and each sample was placed into a collection tube, placed in a box with ice, and shipped to the laboratory where they were frozen at $-20^{\circ} \mathrm{C}$ until processing. The EIA protocol followed the same performed for the sows (section Salivary Cortisol and Enzyme-Linked Immunosorbent Assay).

\section{Data Analysis}

Data were initially tested for normality using the Shapiro-Wilk test. Statistical tests were performed using $\mathrm{R}$ studio software and are specified in the respective figures. Differences with $p<$ 0.05 were considered to be statistically significant and tendency considered was $p<0.10$. The non-parametric Mann-Whitney $U$ test was used in the analysis of sows, as the number of replicates was $<5$ in one of the two groups.

\section{RESULTS}

Non-sham-chewing sows had higher levels of salivary-cortisol concentrations in the morning of days 91 and 92 of gestation (Mann-Whitney $U$-test; $p=0.02 ; Z=2.19$; Figure 1). However, closer to delivery, sham-chewing sows had a tendency toward higher levels of salivary-cortisol on evenings 106 and 107 of gestation (Mann-Whitney $U$-test; $p=0.05 ; Z=-1.90$; Figure 1) than non-sham-chewing sows. In additional, there were a difference in cortisol levels in the placental tissue, in which sham-chewing sows had higher levels of cortisol (MannWhitney $U$-test; $p=0.04 ; Z=2.00$; Figure 2) compared to non-sham-chewing sows. In contrast, there were no differences in the cortisone levels in the placenta between the two groups (Figure 2).

Among piglets, there was no difference in salivary cortisol concentration at weaning (on the morning; $p=0.58 ; Z=0.54$; on the afternoon; $p=0.89 ; Z=0.13$ ) or at 35 days old (on the morning; $p=0.91 ; Z=-0.10$; on the afternoon; $p=0.21$; -1.22 ). Piglets born from sows categorized as sham-chewing did not differ from salivary cortisol (day 28 on the morning; mean = 18.42; $\mathrm{SE}=13.42$; day 28 on the afternoon; mean $=806.24$; $\mathrm{SE}$ $=212.62$; day 35 on the morning; mean $=733.47$; $\mathrm{SE}=254.98$; day 35 on the afternoon; mean $=1608.94$; $\mathrm{SE}=898.37$ ) when compared with piglets from non-sham-chewing sows (day 28 on the morning; mean $=17.00 ; \mathrm{SE}=13.01$; day 28 on the afternoon; mean $=722.48 ; \mathrm{SE}=222.87$; day 35 on the morning; mean $=$ 550.93 ; $\mathrm{SE}=176.67$; day 35 on the afternoon; mean $=561.41$; 

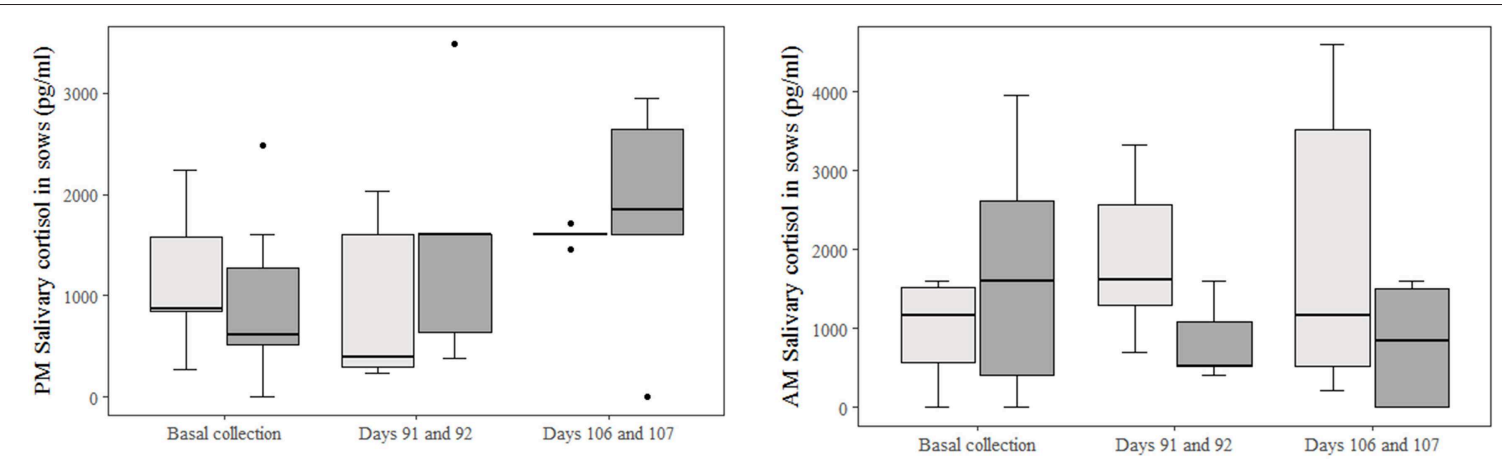
Group
官 Non-sham-chewing
官 Sham-chewing

FIGURE 1 | Sow salivary cortisol concentrations at $6 \mathrm{~h} 00$ and 18h00. Using prenatal behavior observations, sows were categorized as either sham-chewing $(n=4$; dark bars) or non-sham-chewing sows ( $n=7$; light bars). There was a difference on the morning of days 91 and 92 (Mann-Whitney $U$-test; $p=0.001 ; Z=3.20$ ) and a tendency toward greater cortisol levels on the afternoon of days 106 and 107 (Mann-Whitney $U$-test; $p=0.06 ; Z=3.99$ ).

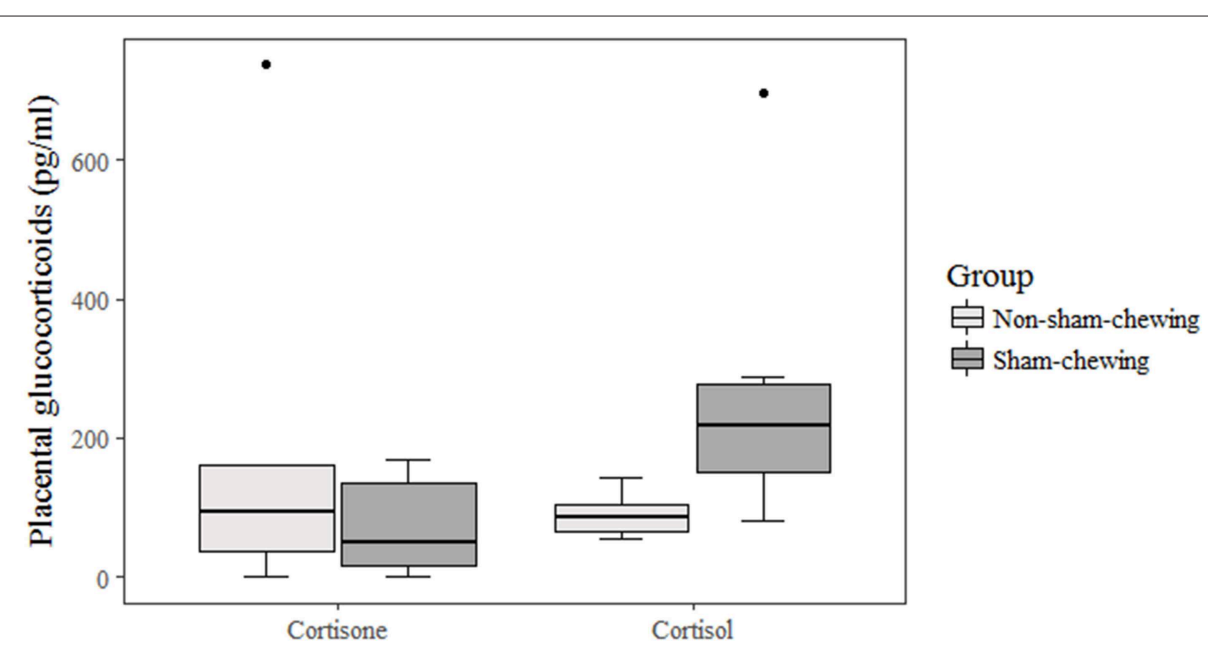

FIGURE 2 | Placental glucocorticoid concentrations. Using prenatal behavior observations, sows were categorized as either sham-chewing ( $n=4$; dark bars) or non-sham-chewing sows ( $n=7$; light bars). Cortisone (Mann-Whitney $U$-test; $p=0.85 ; Z=0.18$ ) and cortisol (Mann-Whitney $U$-test; $p=0.04 ; Z=2.00$ ) concentrations in sows.

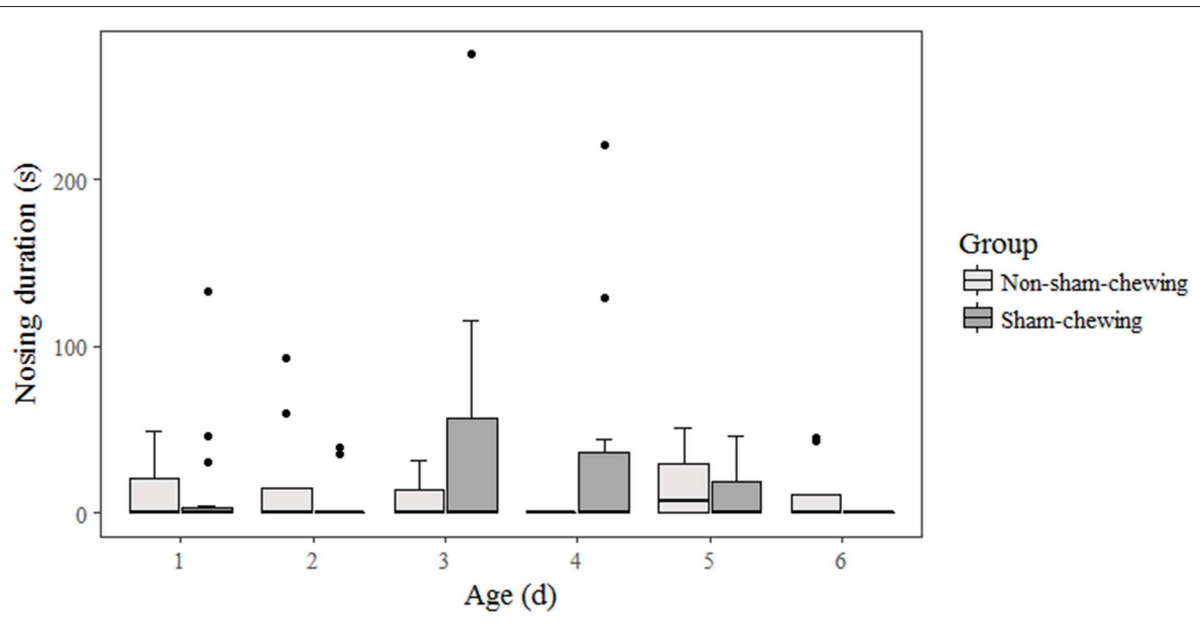

FIGURE 3 | Nosing behavior in piglets. Piglets were from sows that were categorized as either sham-chewing ( $n=4$; dark bars) or non-sham-chewing sows ( $n=7$; light bars). Nosing behavior was higher on day 4 (Mann-Whitney $U$-test; $p=0.03 ; Z=-2.08$ ) and lower on day 6 (Mann-Whitney $U$-test; $p=0.05 ; Z=1.91$ ) in piglets from sows that exhibited stereotypic behavior. 

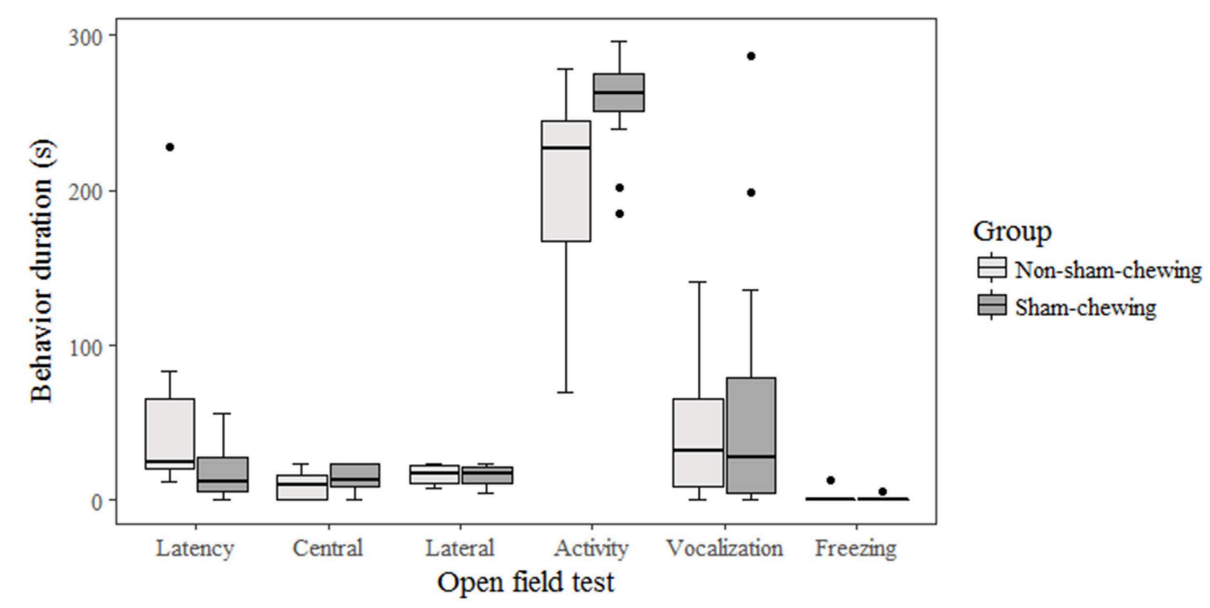

FIGURE 4 | Piglet behaviors during fear and exploratory tests. Piglets were from sows that were categorized as either sham-chewing ( $n=4$; dark bars) or non-sham-chewing sows ( $n=7$; light bars). Piglets from non-sham-chewing sows demonstrated higher latency (Mann-Whitney $U$-test; $p=0.04 ; Z=2.04)$ and less activity (Mann-Whitney $U$-test; $p=0.01 ; Z=-2.38$ ). Latency was the time measured in seconds to start to walk in the arena.

$\mathrm{SE}=213.46)$. The salivary cortisol data is expressed in $\mathrm{pg} / \mathrm{mL}$. In addition, there was no difference in agonistic interactions among piglets from sham-chewing and non-sham-chewing sows $(p>0.05)$. However, in the nosing behavior, piglets from shamchewing sows spent more time performing nosing at the age of 4 days old (Mann-Whitney $U$-test; $p=0.03 ; Z=-2.08$ ) and 6 days old (Figure 3) (Mann-Whitney $U$-test; $p=0.05 ; Z=$ 1.91). During the open field and novel object tests, the differences appeared only during open field tests, in which piglets from nonsham-chewing sows showed higher latency (Mann-Whitney $U$ test; $p=0.04 ; Z=2.04$ ) and less activity (Figure 4) (MannWhitney $U$-test; $p=0.01 ; Z=-2.38$ ). In the novel object test there was no difference among piglets from sham-chewing and non-sham-chewing sows in all measured behaviors (MannWhitney $U$-test, exploring the object, $p=0.49, Z=0.68$; near to object, $p=0.97, Z=0.03$; vocalizations, $p=0.97, Z=-0.03$; freezing, $p=0.58, Z=-0.54$ ).

\section{DISCUSSION}

Based on our data, we concluded that maternal sham-chewing expression could be related to less fear in offspring. Although stereotypies have been studied for decades, attention has not been devoted to the consequences of fetal programming and the long-term impact on future generations.

The higher cortisol concentration in non-sham-chewing sows could be associated with emotionality in the offspring outcomes, considering the potential effects of glucocorticoids on brain development $(21,22,25)$. Although the association between cortisol concentration and stereotypic behavior is a controversial indicator (11), we found that non-sham-chewing sows had greater concentrations on days 91 and 92 of gestation. On days 106 and 107 of gestation, there was a tendency toward a higher concentration in sham-chewing sows. However, these measures were collected close to farrowing (predicted to occur on day 114). In this sense, when delivery is imminent, a cascade starts in offspring cortisol concentration to trigger proper physiological responses (44), and this overlaps with the stereotypy effect.

Moreover, there was a difference in the placental tissue, but only in cortisol concentration, which was higher in shamchewing sows. As this outcome is not congruent with salivary cortisol levels, we can argue that there is difference in $11 \beta \mathrm{HSD}$ enzyme activity, which oxidizes the biologically active form of cortisol in cortisone $(28,29)$. Chronic stressful situations have the potential to inhibit the capacity to upregulate type 2 enzyme activity, and the capacity to adapt placental $11 \beta \mathrm{HSD} 2$ is greatly reduced by previous exposure to chronic stress (30), thus reducing the protection capacity of the placenta. Furthermore, is possible that cortisol is concentrated in the placental tissue instead of crossing and reaching the fetus' brains. In other words, the higher cortisol concentration in sham-chewing sows could result from a placenta holding this glucocorticoid and protecting the brain development in their offspring. We also have some evidence that the epigenome in the limbic system of these piglets is differentially methylated (45).

There was no difference in salivary cortisol concentration in piglets at weaning or at 35 days old. Additionally, there was no difference in aggressive behavior between piglets. However, in nosing behavior (Figure 3), piglets born of sham-chewing sows spent more time performing nosing on day 4 , but not in day 6, and piglets born of non-sham-chewing sows spent more time performing nosing behavior. Nosing and belly nosing is an undesirable piglet-directed behavior expressed after weaning that can sometimes be a trigger for aggressiveness (personal observation) and cause skin lesions in the recipient when persistently performed, as is belly nosing (46). In this study, we considered nosing as any behavior directed for any part of pen mates' body. Once this motor behavior pattern precedes suckling and milk intake, it has been suggested that it may be 
associated with hunger or feeding (42) or even with the artificial early weaning.

In previous studies, there was a negative correlation between suckling behavior in the sow and nosing after weaning (47). Poorquality diet post-weaning and the presence of milk did not affect the development of belly-nosing in piglets weaned between 14 and 18 days old (48). These data support the hypothesis that feeding motivation and hunger is not a causal factor of belly nosing. Moreover, there are differences between nosing (as a piglet-directed behavior) and belly nosing, for which the levels in nosing remain significantly more consistent throughout time and start the first day after weaning, instead of the peak of bellynosing, which appears only in the second week post-weaning and starts to decrease thereafter (48). Another possibility is that the nosing we observed is a stereotypic behavior because it is consistent with the definition of repetitive behavior and appears to have no obvious function (1). As a stereotypic behavior, it can be a strategy to cope with artificial weaning in some piglets.

Moreover, it has been shown that there is a strong genetic basis for the development of stereotypies $(2,49)$. There is not necessarily a congruence between the type of stereotypy exhibited by mothers and offspring (2), although in this sense, it is reasonable to expect stereotypies in offspring from mothers that also performed. However, the genetic component must interact with environmental conditions to trigger this feature in the offspring. Apart from genetic predisposition, the effects of parental behavior cannot be excluded because the offspring passed the first 28 days with their mothers. Maternal behavior affects litter development, which includes behavior and emotionality (50-52).

In the fear tests, piglets from non-sham-chewing sows demonstrated higher latency and less activity, indicating more fear states. Fear is the most common emotion investigated in domestic animals (33) and this emotion is related to welfare since it is a negative emotion. However, evolutionary mechanisms shape emotions to increase fitness, and fear is a reaction to the perception of actual danger to trigger appropriate adaptive responses $(33,53)$. Although pigs in general have an explorative trait in their genome, it is expected that a piglet kept alone in a novel space and that is then faced with a unknown object will experience some level of fear. This response should be adaptive throughout evolution since it is a risk to be alone, exposed to predators, and away from their mates and mother. Nonetheless, it is possible to measure only the indicators of fear once, as every emotion is a subjective state (33).

We have shown in preliminary results that the expression of sham-chewing by the mother affects the offspring's emotionality (45). However, in that experiment, we considered a gradient of sows, in which we divided 28 sows in two groups, from low to high expression in terms of duration. In the open field test, piglets from sows with a high rate of stereotypies walked more in central quadrants and lateral quadrants than piglets from sows with a low rate of stereotypies. Moreover, in the novel object test the offspring from low stereotypy sows vocalized more (45). We demonstrated for the first time that stereotypic behavior expressed by the mother during gestation changes the phenotype of the offspring, in particular their emotionality (45). This outcome can be related to the stress response or reactivity, reflecting stereotypies as an indicator of welfare. In contrast, the present study adopted a focused strategy, in which we considered consistent sham-chewing throughout the days of observation. This approach enabled us to select a desirable profile for answering our question regarding the "thrifty hypothesis." Our data indicate that it is not simply a difference in piglet emotionality, but that piglets from sows that do not exhibit stereotypic behavior exhibit more fear. To our knowledge, these results are the first to indicate that sows exhibiting sham-chewing bear piglets with less fear.

Overall, we consider that it is reasonable to accept that stereotypies are a welfare indicator. When animals are expressing stereotypies, it may indicate that the environment and context in which they are kept is not meeting their needs. However, it is possible that the individuals that are not expressing stereotypies, under the same difficult situation, are experiencing more compromised welfare, as we have shown in our studies investigating the consequences to the offspring.

\section{DATA AVAILABILITY STATEMENT}

The raw data supporting the conclusions of this manuscript will be made available by the authors, without undue reservation, to any qualified researcher.

\section{ETHICS STATEMENT}

This study was approved by the Ethics Committee on Animal Use of the Faculty of Veterinary Medicine and Animal Science, University of São Paulo (CEUA/FMVZ, protocol number 6157201114).

\section{AUTHOR CONTRIBUTIONS}

PT and AZ conceptualized the study and its methodology. PT performed the formal analysis, the project administration, and wrote the original draft. AZ performed the funding acquisition, collected resources, and supervised the study. PT, TB, and LA performed the investigation. PT, TB, LA, and AZ wrote, reviewed, and edited the manuscript.

\section{ACKNOWLEDGMENTS}

The authors are grateful to the Department of Preventive Veterinary Medicine and Animal Health and to the CNPQ for the funding that was provided. They are also grateful to Araporanga Farm for helping with the animals' provision. 


\section{REFERENCES}

1. Mason GJ. Stereotypies and suffering. Behav Process. (1991) 25:103-15. doi: 10.1016/0376-6357(91)90013-P

2. Schwaibold U, Pillay N. Stereotypic behaviour is genetically transmitted in the African striped mouse Rhabdomys pumilio. Appl Anim Behav Sci. (2001) 74:273-80. doi: 10.1016/S0168-1591(01)00169-1

3. Ijichi CL, Collins LM, Elwood RW. Evidence for the role of personality in stereotypy predisposition. Anim Behav. (2013) 85:1145-51. doi: 10.1016/j.anbehav.2013.03.033

4. Joshi S, Pillay N. Association between personality and stereotypic behaviours in the African striped mouse Rhabdomys dilectus. Appl Anim Behav Sci. (2016) 174:154-61. doi: 10.1016/j.applanim.2015.11.021

5. Liu H, Duan H, Wang C. Effects of ambient environmental factors on the stereotypic behaviors of giant pandas (Ailuropoda melanoleuca). PLoS ONE. (2017) 12:e0170167. doi: 10.1371/journal.pone.0170167

6. Mason G, Rushen J. Stereotypic Animal Behaviour : Fundamentals and Applications to Welfare. Wallingford: CABI Pub (2006).

7. Appleby MC, Lawrence AB. Food restriction as a cause of stereotypic behaviour in tethered gilts. Anim Prod. (1987) 45:103-10. doi: 10.1017/S0003356100036680

8. Stewart CL, O'Connell NE, Boyle L. Influence of access to straw provided in racks on the welfare of sows in large dynamic groups. Appl Anim Behav Sci. (2008) 112:235-47. doi: 10.1016/j.applanim.2007.09.006

9. Dantzer R. Stress, stereotypies and welfare. Behav Process. (1991) 25:95-102. doi: 10.1016/0376-6357(91)90012-O

10. Zhang MY, Li X, Zhang XH, Liu HG, Li JH, Bao J. Effects of confinement duration and parity on stereotypic behavioral and physiological responses of pregnant sows. Physiol Behav. (2017) 179:369-76. doi: 10.1016/j.physbeh.2017.07.015

11. Terlouw EMC, Lawrence AB, Ladewig J, De Passille AM, Rushen J, Schouten WGP. Relationship between plasma cortisol and stereotypic activities in pigs. Behav Process. (1991) 25:133-53. doi: 10.1016/0376-6357(91)90016-S

12. Sapolsky RM, Romero LM, Munck AU. How do glucocorticoids influence stress responses? Integrating permissive, suppressive, stimulatory, and preparative actions. Endocr Rev. (2000) 21:55-89. doi: 10.1210/ er.21.1.55

13. Möstl E, Palme R. Hormones as indicators of stress. Domest Anim Endocrinol. (2002) 23:67-74. doi: 10.1016/S0739-7240(02)00146-7

14. Cooper JJ, Nicol CJ. The "coping" hypothesis of stereotypic behaviour: a reply to Rushen. Anim Behav. (1993) 45:616-8. doi: 10.1006/anbe.1993.1072

15. Rushen J. The "coping" hypothesis of stereotypic behaviour. Anim Behav. (1993) 45:613-5. doi: 10.1006/anbe.1993.1071

16. Díez-León M, Bowman J, Bursian S, Filion H, Galicia D, Kanefsky $\mathrm{J}$, et al. Environmentally enriched male mink gain more copulations than stereotypic, barren-reared competitors. PLoS ONE. (2013) 8:e80494. doi: 10.1371/journal.pone.0080494

17. Braastad BO. Effects of prenatal stress on behaviour of offspring $\backslash$ nof laboratory and farmed mammals. Appl Anim Behav Sci. (1998) 61:159-180. doi: 10.1016/S0168-1591(98)00188-9

18. Urakubo A, Jarskog LF, Lieberman JA, Gilmore JH. Prenatal exposure to maternal infection alters cytokine expression in the placenta, amniotic fluid, and fetal brain. Schizophr Res. (2001) 47:27-36. doi: 10.1016/S0920-9964(00)00032-3

19. Darnaudéry M, Maccari S. Epigenetic programming of the stress response in male and female rats by prenatal restraint stress. Brain Res Rev. (2008) 57:571-85. doi: 10.1016/j.brainresrev.2007.11.004

20. Meyer U, Feldon J, Fatemi SH. In-vivo rodent models for the experimental investigation of prenatal immune activation effects in neurodevelopmental brain disorders. Neurosci Biobehav Rev. (2009) 33:1061-79. doi: 10.1016/j.neubiorev.2009.05.001

21. Rutherford KMD, Piastowska-Ciesielska A, Donald RD, Robson SK, Ison $\mathrm{SH}$, Jarvis S, et al. Prenatal stress produces anxiety prone female offspring and impaired maternal behaviour in the domestic pig. Physiol Behav. (2014) 129:255-64. doi: 10.1016/j.physbeh.2014.02.052

22. Baxter EM, Mulligan J, Hall SA, Donbavand JE, Palme R, Aldujaili E, et al. Positive and negative gestational handling influences placental traits and mother-offspring behavior in dairy goats. Physiol Behav. (2016) 157:129-38. doi: 10.1016/j.physbeh.2016.02.001
23. Poletto R, Steibel JP, Siegford JM, Zanella AJ. Effects of early weaning and social isolation on the expression of glucocorticoid and mineralocorticoid receptor and 11beta-hydroxysteroid dehydrogenase 1 and 2 mRNAs in the frontal cortex and hippocampus of piglets. Brain Res. (2006) 1067:36-42. doi: 10.1016/j.brainres.2005.10.001

24. Weinstock M. The long-term behavioural consequences of prenatal stress. Neurosci Biobehav Rev. (2008) 32:1073-86. doi: 10.1016/j.neubiorev.2008.03.002

25. Coulon M, Wellman CL, Marjara IS, Janczak AM, Zanella AJ. Early adverse experience alters dendritic spine density and gene expression in prefrontal cortex and hippocampus in lambs. Psychoneuroendocrinology. (2013) 38:1112. doi: 10.1016/j.psyneuen.2012.10.018

26. Bernardino T, Tatemoto P, Morrone B, Rodrigues PHM, Zanella AJ. Piglets born from sows fed high fibre diets during pregnancy are less aggressive prior to weaning. PLoS ONE. (2016) 11:e0167363. doi: 10.1371/journal.pone.0167363

27. Fowden AL, Valenzuela OA, Vaughan OR, Jellyman JK, Forhead AJ. Glucocorticoid programming of intrauterine development. Domest Anim Endocrinol. (2016) 56:S121-32. doi: 10.1016/j.domaniend.2016.02.014

28. Jansson T, Powell TL. Role of the placenta in fetal programming: underlying mechanisms and potential interventional approaches. Clin Sci. (2007) 113:113. doi: 10.1042/CS20060339

29. Seckl JR. Prenatal glucocorticoids and long-term programming. Eur J Endocrinol. (2004) 151(Suppl.):U49-62. doi: 10.1530/eje.0.151u049

30. Welberg LAM, Thrivikraman KV, Plotsky PM. Chronic maternal stress inhibits the capacity to up-regulate placental 11-beta-hydroxysteroid dehydrogenase type 2 activity. J Endocrinol. (2005) 186:R7-12. doi: 10.1677/joe.1.06374

31. Abe H, Hidaka N, Kawagoe C, Odagiri K, Watanabe Y, Ikeda T, et al. Prenatal psychological stress causes higher emotionality, depression-like behavior, and elevated activity in the hypothalamo-pituitary-adrenal axis. Neurosci Res. (2007) 59:145-51. doi: 10.1016/j.neures.2007.06.1465

32. Nolvi S, Karlsson L, Bridgett DJ, Korja R, Huizink AC, Kataja E-L, et al. Maternal prenatal stress and infant emotional reactivity six months postpartum. J Affect Disord. (2016) 199:163-70. doi: 10.1016/j.jad.2016.04.020

33. Forkman B, Boissy A, Meunier-Salaün MC, Canali E, Jones RB. A critical review of fear tests used on cattle, pigs, sheep, poultry and horses. Physiol Behav. (2007) 92:340-74. doi: 10.1016/j.physbeh.2007.03.016

34. Donald RD, Healy SD, Lawrence AB, Rutherford KMD. Emotionality in growing pigs: Is the open field a valid test? Physiol Behav. (2011) 104:906-13. doi: 10.1016/j.physbeh.2011.05.031

35. Rutherford KMD, Donald RD, Lawrence AB, Wemelsfelder F. Qualitative behavioural assessment of emotionality in pigs. Appl Anim Behav Sci. (2012) 139:218-24. doi: 10.1016/j.applanim.2012.04.004

36. Fijn L, Antonides A, Aalderink D, Nordquist RE, van der Staay FJ. Does litter size affect emotional reactivity, spatial learning and memory in piglets? (2016) 178:23-31. doi: 10.1016/j.applanim.2016.02.011

37. Zonderland JJ, De Leeuw JA, Nolten C, Spoolder HAM. Assessing long-term behavioural effects of feeding motivation in group-housed pregnant sows; what, when and how to observe. Appl Anim Behav Sci. (2004) 87:15-30. doi: 10.1016/j.applanim.2003.12.009

38. Cooper TR, Trunkfield HR, Zanella AJ, Booth WD. An enzyme-linked immunosorbent assay for cortisol in the saliva of man and domestic farm animals. J Endocrinol. (1989) 123:R13-6. doi: 10.1677/joe.0.123R013

39. Bradford MM. A rapid and sensitive method for quantitation of microgram quantities of protein utilizing the principle of protein-dye-binding. Anal Biochem. (1976) 72:248-54. doi: 10.1016/0003-2697(76)90527-3

40. Colson V, Orgeur P, Courboulay V, Dantec S, Foury A, Mormède P. Grouping piglets by sex at weaning reduces aggressive behaviour. Appl Anim Behav Sci. (2006) 97:152-71. doi: 10.1016/j.applanim.200 5.07.006

41. Jensen P, Yngvesson J. Aggression between unacquainted pigs-sequential assessment and effects of familiarity and weight. Appl Anim Behav Sci. (1998) 58:49-61. doi: 10.1016/S0168-1591(97)00097-X

42. Fraser D. The behaviour of growing pigs during experimental social encounters. J Agric Sci. (1974) 82:147-63. doi: 10.1017/S0021859600050322

43. Zupan M, Framstad T, Zanella AJ. Behaviour, heart rate, and heart rate variability in pigs exposed to novelty. Rev Bras Zootecnia. (2016) 45:121-9. doi: 10.1590/S1806-92902016000300006 
44. Challis JRG, Sloboda D, Matthews SG, Holloway A, Alfaidy N, Patel FA, et al. The fetal placental hypothalamic-pituitary-adrenal (HPA) axis, parturition and post natal health. Mol Cell Endocrinol. (2001) 185:135-44. doi: 10.1016/S0303-7207(01)00624-4

45. Tatemoto P, Bernardino T, Morrone B, Queiroz MR, Zanella AJ. Stereotypic behaviour in sows modifies is related with emotionality changes in the offspring. Front Vet Sci. (2019). [Epub ahead of print].

46. Gardner JM, De Lange CFM, Widowski TM. Belly-nosing in early-weaned piglets is not influenced by diet quality or the presence of milk in the diet. $J$ Anim Sci. (2001) 79:73-80. doi: 10.2527/2001.79173x

47. Torrey S, Widowski TM. Is belly nosing redirected suckling behaviour? Appl Anim Behav Sci. (2006) 101:288-304. doi: 10.1016/j.applanim.2006.02.009

48. Gardner JM, Duncan IJH, Widowski TM. Effects of social "stressors" on belly-nosing behaviour in early-weaned piglets: Is belly-nosing an indicator of stress? Appl Anim Behav Sci. (2001) 74:135-52. doi: 10.1016/S0168-1591(01)00158-7

49. Schoenecker B, Heller K. Indication of a genetic basis of stereotypies in laboratory-bred bank voles (Clethrionomys glareolus). Appl Anim Behav Sci. (2000) 68:339-47. doi: 10.1016/S0168-1591(00) 00114-3

50. Francis D, Diorio J, Liu D, Meaney MJ. Nongenomic transmission across generations of maternal behavior and stress responses in the rat. Science. (1999) 286:1155-8. doi: 10.1126/science.286.5442.1155
51. Pedersen CA, Vadlamudi S, Boccia ML, Moy SS. Variations in maternal behavior in C57BL/6J mice: behavioral comparisons between adult offspring of high and low pup-licking mothers. Front Psychiatry. (2011) 2:42. doi: 10.3389/fpsyt.2011.00042

52. Borrow AP, Cameron NM. Maternal care and affective behavior in female offspring: Implication of the neurosteroid/GABAergic system. Psychoneuroendocrinology. (2017) 76:29-37. doi: 10.1016/ j.psyneuen.2016.10.028

53. Dunsmoor JE, Paz R. Fear generalization and anxiety: behavioral and neural mechanisms. Biol Psychiatry. (2015) 78:336-43. doi: 10.1016/j.biopsych.2015.04.010

Conflict of Interest: The authors declare that the research was conducted in the absence of any commercial or financial relationships that could be construed as a potential conflict of interest.

Copyright (c) 2019 Tatemoto, Bernardino, Alves and Zanella. This is an open-access article distributed under the terms of the Creative Commons Attribution License (CC $B Y)$. The use, distribution or reproduction in other forums is permitted, provided the original author(s) and the copyright owner(s) are credited and that the original publication in this journal is cited, in accordance with accepted academic practice. No use, distribution or reproduction is permitted which does not comply with these terms. 